\title{
Taiwan Automated Telescope Network
}

\section{Dean-Yi Chou, ${ }^{1}$ Ming-Tsung Sun, ${ }^{2}$ Javier Fernandez Fernandez, ${ }^{1}$ Li-Han Wang, ${ }^{1}$ Antonio Jimenez, ${ }^{3}$ Alexander Serebryanskiy, ${ }^{4}$ and Shuhrat Ehgamberdiev ${ }^{4}$}

\author{
${ }^{1}$ Department of Physics, National Tsing Hua University, Hsinchu 30013, Taiwan \\ ${ }^{2}$ Department of Mechanical Engineering, Chang-Gung University, Kwei-San 33302, Taiwan \\ ${ }^{3}$ Instituto de Astrofisica de Canarias, 38205 La Laguna, Spain \\ ${ }^{4}$ Ulugh Beg Astronomical Institute, 100052 Tashkent, Uzbekistan
}

Correspondence should be addressed to Dean-Yi Chou, chou@phys.nthu.edu.tw

Received 15 June 2009; Accepted 5 October 2009

Academic Editor: Taro Kotani

Copyright (C) 2010 Dean-Yi Chou et al. This is an open access article distributed under the Creative Commons Attribution License, which permits unrestricted use, distribution, and reproduction in any medium, provided the original work is properly cited.

A global network of small automated telescopes, the Taiwan Automated Telescope (TAT) network, dedicated to photometric measurements of stellar pulsations, is under construction. Two telescopes have been installed in Teide Observatory, Tenerife, Spain and Maidanak Observatory, Uzbekistan. The third telescope will be installed at Mauna Loa Observatory, Hawaii, USA. Each system uses a 9 -cm Maksutov-type telescope. The effective focal length is $225 \mathrm{~cm}$, corresponding to an $f$-ratio of 25 . The field of view is 0.62 degree square. The images are taken with a 16-bit $1024 \times 1024$ CCD camera. The telescope is equipped with UBVRI filters. Each telescope is fully automated. The telescope can be operated either interactively or fully automatically. In the interactive mode, it can be controlled through the Internet. In the fully automatic mode, the telescope operates with preset parameters without any human care, including taking dark frames and flat frames. The network can also be used for studies that require continuous observations for selected objects.

\section{Introduction}

The global ground-based telescope network is a way to continuously monitor selected objects. The Hungarian Automated Telescope (HAT) Network, dedicated to the extrasolar planet search, so far has two sites [1]. The Stellar Oscillations Network Group (SONG) is a ground-based network dedicated to measurements of stellar pulsation velocity. It plans to build eight identical telescopes around the globe [2]. Here we introduce the Taiwan Automated Telescope (TAT) Network, which is a ground-based network dedicated to photometric measurements of stellar pulsations to study the structure and evolution of stars. The network will consist of several identical robotic optical telescopes at appropriate longitudes around the globe to continuously measure stellar pulsations with a high duty cycle. The headquarter of the network is located at the Physics Building of National Tsing Hua University, Taiwan, where the electronic devices and software are designed and built. The mechanical devices are designed and built in ChangGung University. So far two telescopes have been installed at
Teide Observatory $\left(28^{\circ} 18^{\prime} \mathrm{N}, 16^{\circ} 31^{\prime} \mathrm{W}\right)$, Tenerife, Spain and Maidanak Observatory $\left(38^{\circ} 41^{\prime} \mathrm{N}, 66^{\circ} 56^{\prime} \mathrm{E}\right)$, Uzbekistan. Figure 2 shows the picture of the telescope at Maidanak. The third telescope is planned to be installed at Mauna Loa Observatory $\left(19^{\circ} 32^{\prime} \mathrm{N}, 155^{\circ} 35^{\prime} \mathrm{W}\right)$, Hawaii, USA. The search for the fourth site is underway. Each telescope is fully automated. The telescope can be operated either interactively or fully automatically. In the interactive mode, it can be controlled through the Internet. In the fully automatic mode, the telescope operates with preset parameters without any human care, including taking dark frames and flat frames. The telescope can measure 10-magnitude stars with an error of about 2-3 mmag. The long-term goal of the project is to upgrade the telescopes to ones with a larger aperture.

\section{Hardware}

The overall structure of the automated telescope is described by a system block diagram shown in Figure 1. The major components are briefly described as follows. 


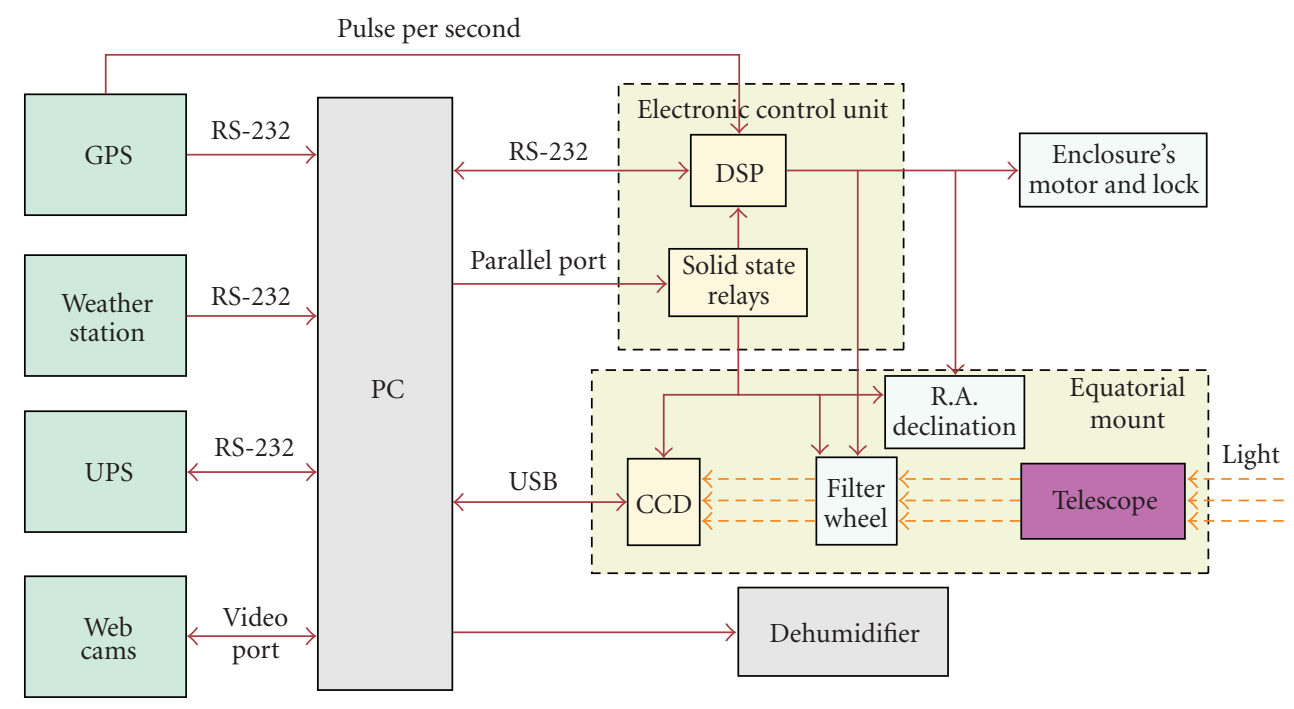

FIGURE 1: Block diagram of the telescope system.

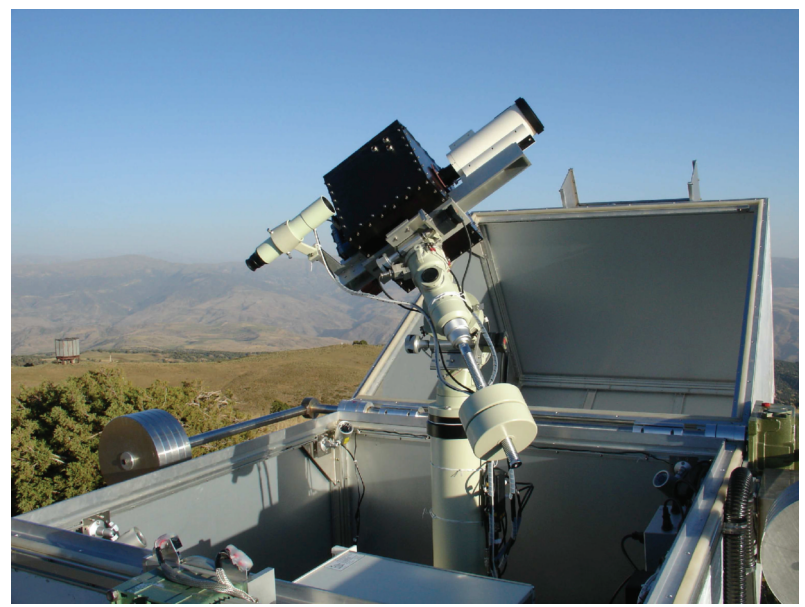

Figure 2: Telescope at the Maidanak Observatory, Uzbekistan.

2.1. Computer. The whole system is controlled by a Linuxbased industrial personal computer, which allows us to install several interface cards. The computer uses an RAID-1 system to avoid loss of data and for a quick recovery. A digital signal processor (DSP) is used as an interface between the computer and some hardware devices, such as filter wheel, stepping motors, limit switches, and enclosure.

2.2. Optics and $C C D$. Each system uses a $9 \mathrm{~cm}$ (3.5-inch) ruggedized Questar telescope (Maksutov type). The focus of the telescope can be locked with six screws on three rods attached to the main mirror. The field of view can vary by changing the separation between the telescope and the CCD camera. The images are taken with a 16-bit $1024 \times 1024$ aircooled CCD camera, using a Kodak KAF-1001E chip, made by Apogee. The dimension of the chip is $2.45 \times 2.45 \mathrm{~cm}$. The field of view is currently set to 0.62 degree square.
The effective focal length is $225 \mathrm{~cm}$, corresponding to an $f$ ratio of 25 . The telescope and the CCD are mounted on an aluminum beam, and their separation can be changed for the focal length adjustment. A filter wheel with UBVRI filters, covered with a case, is mounted between the telescope and the CCD. The filter wheel is driven by a stepping motor which is controlled by the computer through the DSP.

2.3. Telescope Tracking. The telescope is mounted on a German-type equatorial mount, made by Takahashi. The original two Takahashi step motors of right ascension (RA) and declination (DEC) are replaced by two five-phase stepping motors made by Oriental Motor. The two stepping motors are controlled by the DSP, which is in turn controlled by the computer. Two photo interrupters are used for the reference position of the RA and DEC. The target position is computed relative to this reference position, and the telescope moves to the reference position before moving to the target position [3]. Limit switches are used to prevent the telescope from moving out of range. The telescope tracking is corrected by cross-correlating the current image with a reference image. This correction is carried out after each exposure.

2.4. Enclosure. A rectangular-shape enclosure is used to house the telescope and all equipments except the external weather sensors and webcams and GPS antenna. A rectangular-shape enclosure instead of a conventional dome is used because it can provide a better seal. A dehumidifier is installed inside the enclosure to reduce humidity during nonobserving periods. A heater is used to ensure the function of the dehumidifier and UPS batteries in the winter.

2.5. Monitoring Webcams. Eight to ten webcams are used to monitor the status of the telescope and other equipments. Some of the webcams are installed inside the enclosure. The webcams are equipped with infrared light source for 
night monitoring. They are automatically turned off during observations to avoid light pollution. A couple of wide-angle webcams of $150^{\circ}$ point to the sky to monitor the sky quality. The video streams from the webcams can be viewed on the webpage of each station.

2.6. GPS. A GPS is used to provide the computer with the precise time/date. It also provides the DSP with the signal of pulse per sec (PPS) because the DSP needs an accurate time reference.

2.7. Weather Station. A weather station is set up near the telescope to provide basic weather data for the autoobserving software to determine whether the observation can start. The weather data are also used to determine the emergency stop during observations. The observing criterion could vary from site to site. It is currently set as the wind speed less than $20 \mathrm{~m} / \mathrm{s}$ and the relative humidity lower than $70 \%$ for Teide and Maidanak sites.

2.8. Uninterrupted Power Supply. An uninterrupted power supply (UPS) of $1500 \mathrm{VA}$ is used to protect the computer and other electrical equipments. The UPS is connected to the computer through a serial port. The UPS will shut down the computer in case of persistent power failure and turn it on again when the power is resumed.

\section{Software}

3.1. Automatic Control. Each telescope is fully automated. The telescope can be operated either interactively or fully automatically. In the interactive mode, it can be controlled through the Internet. The observer, logging in the computer, can control each component of the system through a userfriendly command menu.

In the fully automatic mode, the telescope operates with a set of preset parameters without any human care. All preset parameters are written in a file, checked by the computer every minute. The parameters include the beginning and end of the observing period, the coordinates of the target, the exposure time, the CCD temperature, and the filter wheel position. The file can contain multiday parameters. When it reaches the preset beginning of observing time and the weather criterion is satisfied, the enclosure is unlocked and opened. The telescope moves to the target star and tracks it. The CCD takes images at the preset rate. At the end of observations, the telescope moves back to the reference position, and the enclosure is closed and locked. If weather conditions change and fail the observing criterion during the observation, the observation will be stopped and the enclosure will be closed immediately. The automatic observing procedure also includes taking dark frames and twilight flat frames before and after observations. The observed images are stored in the hard disk drives and transferred back to the headquarter through the Internet.

3.2. Website. Each station has its own website providing the status and information of the system. Observers can

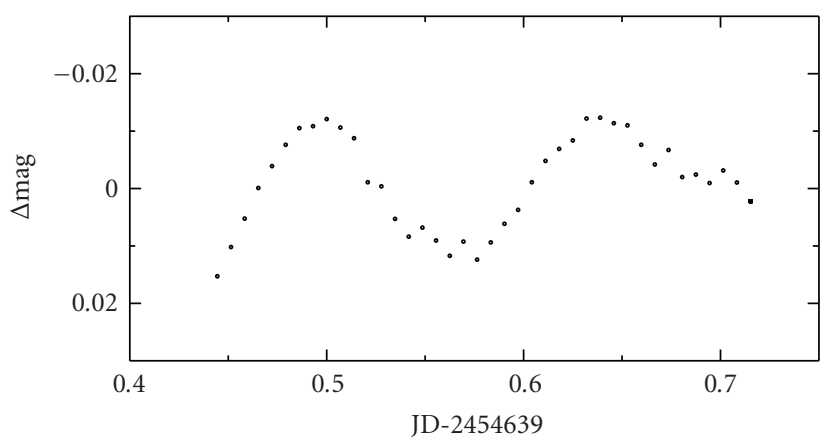

Figure 3: Light curves of HD 163032 from data taken on 18 May 2007 at Teide. The exposure time was 560 seconds. No filter was used in this observation. HD 163032 is an A5 star with $m_{V}=8$ [10].

monitor the status and obtain information from the website. It includes the life images from all webcams, the history of weather conditions, and the history of UPS status.

\section{Scientific Goals and Preliminary Results}

The Taiwan Automated Telescope Network will be dedicated to observing stellar pulsations for the study the stellar structure and evolution, although it could be used for other studies that require continuous observations for selected objects. Major information used to study the properties of pulsating stars is the frequency of pulsating modes [4-8]. The advantage of the TAT Network is that it can observe a target star for a long period with high duty cycle. This would increase the $\mathrm{S} / \mathrm{N}$ and the spectral resolution which would increase accuracy in determining mode frequencies. The long data set provides the opportunity to study temporal variations of mode parameters, such as amplitude, frequency, and line width $[6,9]$. The long data set could also be used to search for long-period modes, such as $g$ modes [6]. The data will be available to the community after a certain period.

Although the aperture of the TAT is small, there are still many bright variable stars which could be studied with the TAT. The telescope can measured 10-magnitude stars with an error of about 2-3 mmag with 10-minute exposure. Figure 3 shows an example of the light curve of HD163032 derived from the data taken with the TAT at Teide. It should be mentioned that combining data taken at different sites will be a challenge in data analysis.

\section{Acknowledgment}

The authors are supported by NSC of ROC under Grant NSC-96-2112-M-007-034-MY3.

\section{References}

[1] G. Á. Bakos, J. Lázár, I. Papp, P. Sári, and E. M. Green, “System description and first light curves of the Hungarian automated telescope, an autonomous observatory for variability search," Publications of the Astronomical Society of the Pacific, vol. 114, no. 799, pp. 974-987, 2002. 
[2] F. Grundahl, T. Arentoft, J. Christensen-Dalsgaard, S. Frandsen, H. Kjeldsen, and P. K. Rasmussen, "Stellar Oscillations Network Group —SONG," Journal of Physics: Conference Series, vol. 118, no. 1, Article ID 012041, 2008.

[3] J. Meeus, Astronomical Algorithms, Willmann-Bell, Richmond, Va, USA, 2nd edition, 1998.

[4] C. Aerts, J. Christensen-Dalsgaard, M. Cunha, and D. W. Kurtz, "The current status of asteroseismology," Solar Physics, vol. 251, no. 1-2, pp. 3-20, 2008.

[5] T. M. Brown and R. L. Gilliland, "Asteroseismology," Annual Review of Astronomy and Astrophysics, vol. 32, no. 1, pp. 3782, 1994.

[6] A. Gautschy and H. Saio, "Stellar pulsations across the HR diagram: Part II," Annual Review of Astronomy and Astrophysics, vol. 34, no. 1, pp. 551-606, 1996.

[7] J. Christensen-Dalsgaard, "Asteroseismology," Astrophysics and Space Science, vol. 261, no. 1-4, pp. 1-12, 1998.

[8] D. W. Kurtz, "Asteroseismology: from dream to reality," Solar Physics, vol. 220, no. 2, pp. 123-135, 2004.

[9] P. Moskalik, "Modulation of amplitudes in oscillating stars due to resonant mode coupling," Acta Astronomica, vol. 35, pp. 229-254, 1985.

[10] ESA, "The Hipparcos and Tycho Catalogues," ESA SP 1200, ESA, Noordwijk, The Netherlands, 1997. 

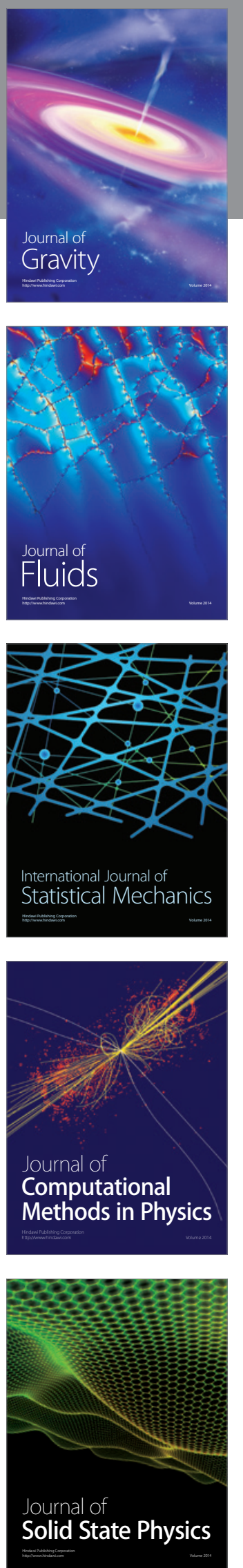

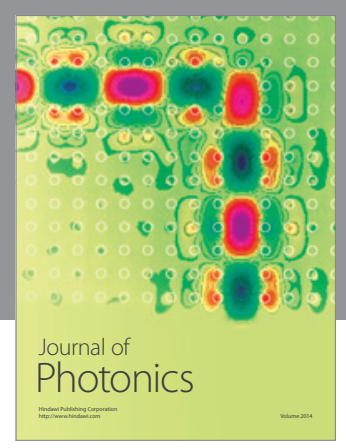

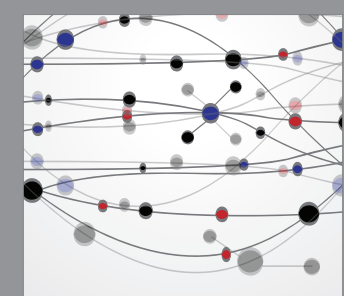

The Scientific World Journal
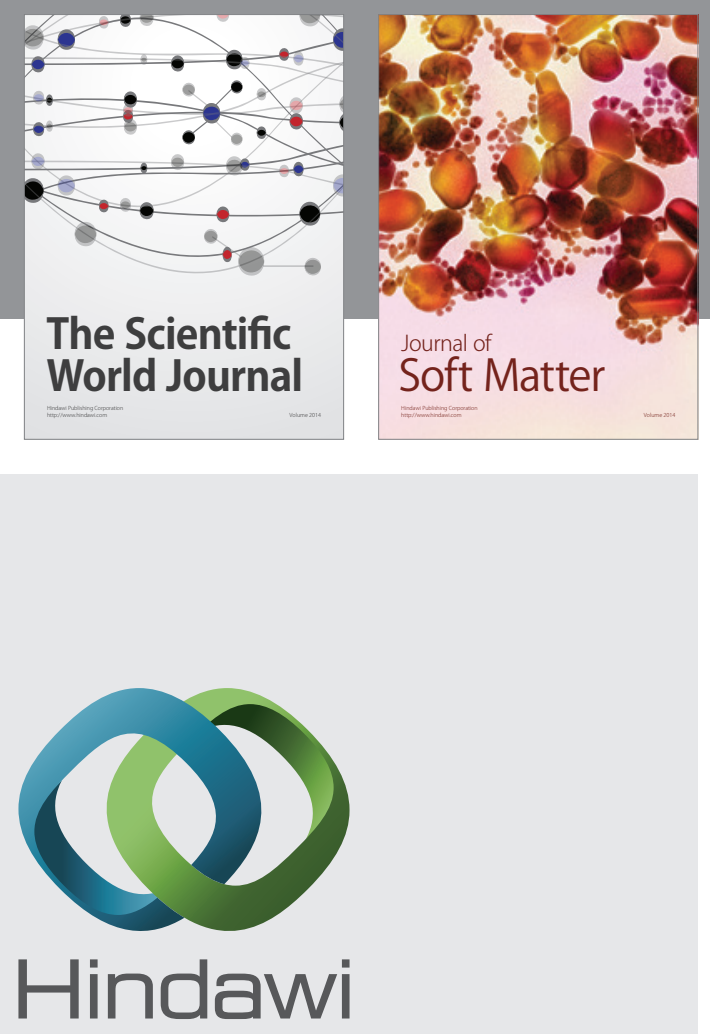

Submit your manuscripts at

http://www.hindawi.com
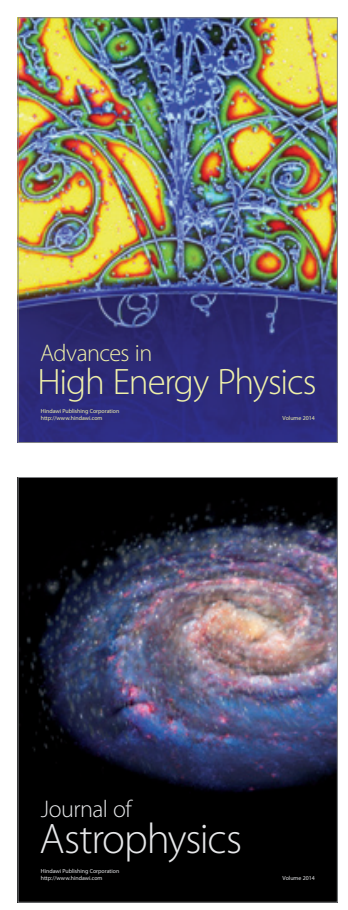
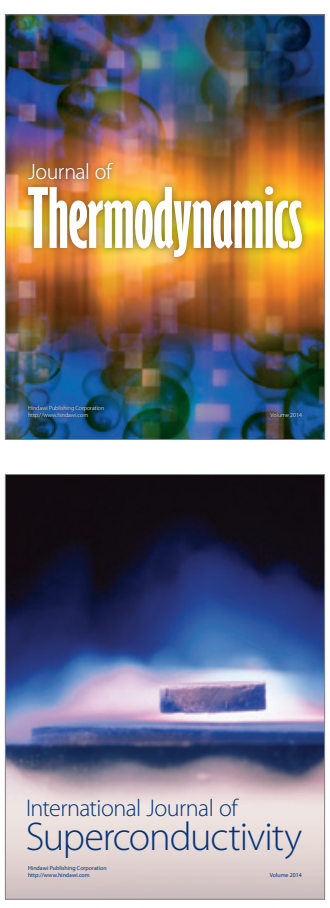
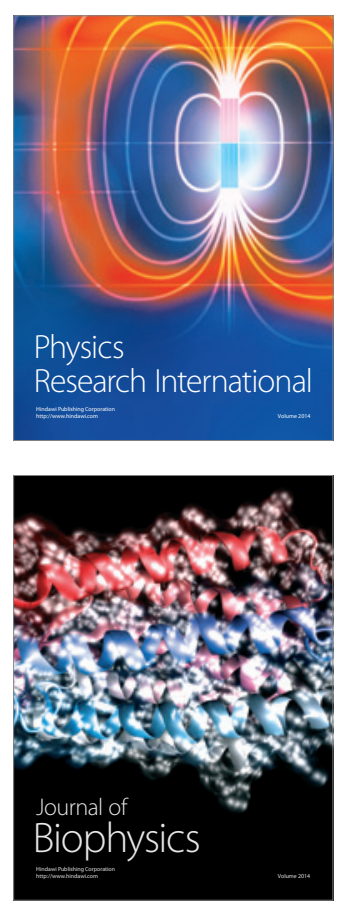
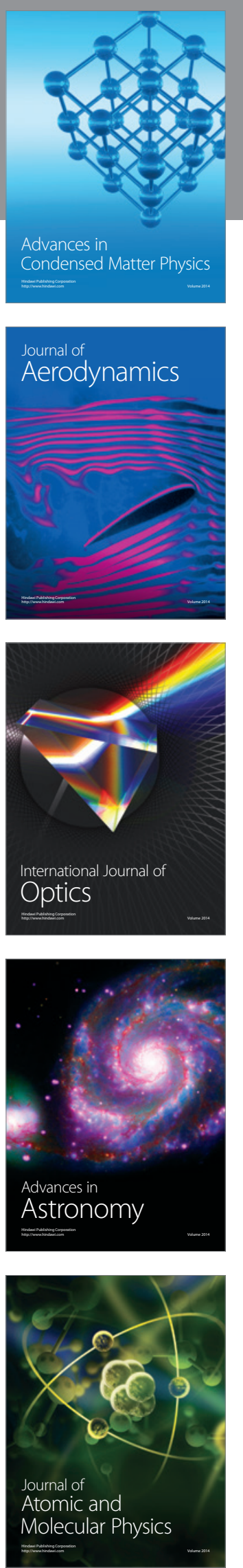